\title{
Erratum to: Attention training for infants at familial risk of ADHD (INTERSTAARS): study protocol for a randomised controlled trial
}

Amy Goodwin ${ }^{1 *}$, Simona Salomone ${ }^{2}$, Patrick Bolton 1,3,4 ${ }^{1}$ Tony Charman ${ }^{5}$, Emily J.H. Jones², Luke Mason², Andrew Pickles ${ }^{6}$, Emily Robinson ${ }^{6}$, Tim Smith ${ }^{7}$ Edmund J.S. Sonuga-Barke ${ }^{8,9}$, Sam Wass ${ }^{10,11}$ and Mark H. Johnson ${ }^{2}$

\section{Erratum}

The original publication [1] misses one author. The author details can be found below.

Dr. Luke Mason, Centre for Brain and Cognitive Development, Birkbeck, University of London, London, UK.

\begin{abstract}
Author details
'Department of Child and Adolescent Psychiatry, Institute of Psychiatry, Psychology and Neuroscience (IoPPN), King's College London, London, UK. ${ }^{2}$ Centre for Brain and Cognitive Development, Birkbeck, University of London, London, UK. ${ }^{3} \mathrm{MRC}$ Social, Genetic and Developmental Psychiatry Centre, IOPPN, King's College London, London, UK. ${ }^{4} \mathrm{NIHR}$ Biomedical Research Centre at South London and Maudsley NHS Trust, London, UK. ${ }^{5}$ Department of Psychology, loPPN, King's College London, London, UK. 'Department of Biostatistics, loPPN, King's College London, London, UK. 'Department of Psychological Sciences, Birkbeck, University of London, London, UK.

${ }^{8}$ Department of Psychology, University of Southampton, Southampton, UK. ${ }^{9}$ Department of Experimental Clinical and Health Psychology, Ghent University, Ghent, Belgium. ${ }^{10}$ School of Psychology, University of East London, London, UK. ${ }^{11}$ Department of Psychology, University of Cambridge, Cambridge, UK.
\end{abstract}

Published online: 11 September 2017

\section{Reference}

1. Goodwin A. Attention training for infants at familial risk of ADHD

(INTERSTAARS): study protocol for a randomised controlled trial. Trials. 2016;

17:608. https://doi.org/10.1186/s13063-016-1727-0

\footnotetext{
* Correspondence: amy.goodwin@kcl.ac.uk

'Department of Child and Adolescent Psychiatry, Institute of Psychiatry,

Psychology and Neuroscience (IoPPN), King's College London, London, UK

Full list of author information is available at the end of the article
} 